\title{
Nuss Procedure: Decrease in Bar Movement Requiring Reoperation with Primary Placement of Two Bars
}

\author{
Amy B. Stanfill, MD, Nerina DiSomma, Steven M. Henriques, MD, \\ Lizabeth J. Wallace, MS, Ravindra K. Vegunta, MBBS, and Richard H. Pearl, MD
}

\begin{abstract}
Background: The Nuss procedure, first reported in 1998, is currently the treatment of choice for pectus excavatum. The most significant bar-related complication documented is bar movement, requiring reoperation in $3.4 \%-27 \%$ of reports. Our report compares the initial placement of one Nuss bar versus two to prevent bar displacement.

Subjects and Methods: An Institutional Review Board-approved, retrospective chart review was performed of all Nuss procedures performed from November 2000 through February 2010. Since November 2006, all initial Nuss procedures were started with the intent of placing two bars. Haller index, patient demographics, duration of surgery, length of stay, postoperative wound infections, and bar movement requiring reoperation were collected and compared for the one-bar versus two-bar patient populations.

Results: In total, 85 Nuss procedures (58 with one-bar and 27 with two-bar primary Nuss procedures) were analyzed. Two attending pediatric surgeons performed all the procedures. Reoperation for bar movement when one bar was initially placed occurred in 9 patients $(15.5 \%)$. No patients with initial placement of two bars required operative revision for a displaced Nuss bar (15.5\% versus $0 \%, P=.05)$. Patient age and Haller index were not statistically different between groups.

Conclusions: Our data demonstrate improved bar stability with no reoperative intervention when pectus excavatum is initially repaired with two Nuss bars. Primary placement of two bars has now become standard practice in our institution for correction of pectus excavatum by the Nuss procedure and would be our recommendation for consideration by other centers.
\end{abstract}

\section{Introduction}

$\mathbf{T}$ HE NUSS PROCEDURE, first described in $1998,{ }^{1}$ is now considered the treatment of choice for the repair of pectus excavatum in specialized pediatric centers. This anomaly of the chest wall occurs in 1 of 1000 children in the United States and accounts for more than $87 \%$ of the congenital chest wall deformities. ${ }^{2,3}$ Minimally invasive repair of this defect by the Nuss procedure has several unique and, at times, emergency complications such as internal mammary artery laceration, pericardial effusion, immediate recurrence of the defect after bar removal, ${ }^{3}$ pericarditis, ${ }^{4}$ incarcerated diaphragmatic hernia, ${ }^{5}$ and cardiac perforation. ${ }^{6}$

Bar-specific complications have also been reported and are extremely troublesome to both surgeon and patient. Bar movement (flipped, rotated, or slipped) has been reported in $3.4 \%^{7}$ to $27 \%{ }^{8}$ of cases in selected patient populations. Several modifications of the original procedure have been undertaken in an attempt to minimize this phenomenon. Our institution sought to reduce bar movement by placement of two Nuss bars at the initial operation.

\section{Subjects and Methods}

An Institutional Review Board-approved, retrospective chart review was performed on all Nuss procedures performed in our institution from November 2000 through February 2010. Haller index, patient demographics, length of surgery, length of stay, and bar-related complicationsspecifically, bar movement requiring reoperation-were collected and compared for the one-bar versus two-bar patient populations. Starting in November 2006, all patients had two bars placed at initial operation. Data were analyzed using SPSS version 18 for Windows.

Department of Pediatric Surgery, Children's Hospital of Illinois, University of Illinois College of Medicine at Peoria, Peoria, Illinois. 


\section{Surgical technique}

Two attending pediatric surgeons performed all of the procedures. After induction of anesthesia and thoracic epidural placement by the anesthesia service, the patients were placed in the supine position with arms abducted at the shoulder no more than $90^{\circ}$. Patients received cefazolin sodium (Ancef ${ }^{\circledR}$; GlaxoSmithKline, London, UK) at $25 \mathrm{mg} / \mathrm{kg}$ prior to surgical incision. Right pleural cavity thoracoscopic guidance was utilized throughout the procedure. The deepest point of the pectus depression was identified, as were the highest points of the rib cage on either side of the defect (crest). One rib space above and one rib space below the deepest point of depression were identified. Bilateral 6-cm incisions lateral to the deepest point of depression were created in the midaxillary lines, subcutaneous pockets were created, and the right pleural cavity was penetrated at the crest point at the superior rib space. Using the tunneling device, the mediastinum was traversed, the left chest was entered, and the upper crest point was penetrated to exit the chest. Bar sizing and preliminary shaping were performed prior to entering the chest cavity, and the bar was molded into shape. If necessary, reshaping of the bar was performed after the bar was placed. The Nuss bar (Walter Lorenz Surgical, Jacksonville, FL) was then brought across from the right to the left chest and flipped into position. The procedure was then repeated for the inferior rib space. One lateral stabilizer was placed on each bar, on opposite sides of the chest, depending on which gave the best fit. The stabilizers were wired to the bars using \#6 surgical steel wire (A and E Medical Corp., Farmingdale, NJ). The bar and stabilizers were then affixed to the chest wall musculature and fascia using a series of absorbable sutures. Retained pleural air was evacuated via the trocar site tubing using a water bath after the chest wall incisions had been closed in layers. Intraoperative chest radiograph was taken prior to the patient's emergence from anesthesia to ensure evacuation of the pneumothorax and document placement of the bars.
Bar removal was performed by opening the bilateral lateral chest wall incisions. The stabilizing bars were identified preoperatively by reviewing the prior chest radiographs. The dissection was limited toward the superior or inferior stabilizing bar, depending on the location of the stabilizer as identified on the imaging study. Removal of the stabilizers is similar in technique to removal when only one bar and stabilizer are placed and will not be further detailed herein.

\section{Results}

In total, 85 Nuss procedures were analyzed: 58 with one bar placed and 27 with two bars placed. In the one-bar group, 9 patients required reoperation specifically for bar movement. This is a significant difference compared with the two-bar group (9 patients [15.5\%] versus 0 patients; $P=.05$ ). Bar movement was equally distributed across all years (20002006) until November 2006, when our standard procedure included primary placement of two bars.

Mean age did not differ significantly between groups (14.6 years in the one-bar group versus 16.6 years in the two-bar group; $P=.88$ ). The Haller index was similar between groups (one-bar index 4.61 versus two-bar index $4.40 ; P=.57$ ). Average case time (1.6 hours [ 1 hour 36 minutes] for one bar versus 1.9 hours [ 1 hours 54 minutes] for two bars; $P=.002$ ) and total operating room time ( 2 hours 48 minutes for one bar versus 3 hours 12 minutes for two bars; $P=.001$ ) were both significantly longer for the two-bar group. Hospital length of stay in days was not significantly different between groups (4.2 days for one bar versus 3.8 days for two bars; $P=.30$ ). Wound infection rates for the two groups were not significantly different $(6.9 \%$ for one bar versus $0 \%$ for two bars; $P=.30)$. (See Figure 1 for complete results.)

\section{Discussion}

The introduction of the Nuss procedure marked a major advancement in repair of congenital chest wall deformities.

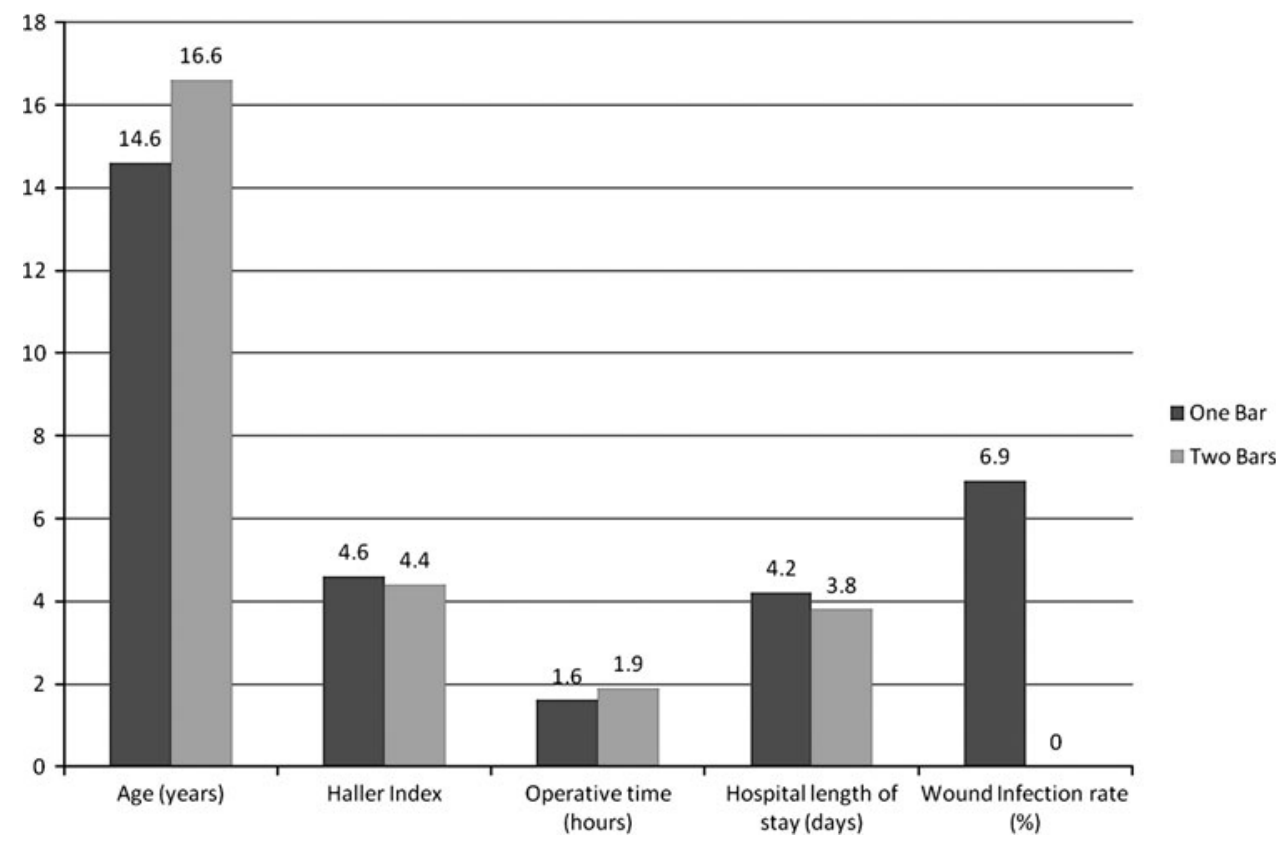

FIG. 1. Differences in outcome measures between the one-bar and two-bar groups. 
However, this procedure does have its own unique set of complications. One of the most troubling and common complications is movement of the Nuss bars causing recurrence of the defect or an inadequate repair, necessitating revision. These bar movement episodes have been reported in $3.4 \%{ }^{7}$ to $27 \%{ }^{8}$ of patients and include bar rotation, flipping, or sliding.

Multiple variations in the initially reported procedure have been undertaken to minimize this complication. Addition of lateral stabilizing endplates has been shown to decrease bar movement from $14.9 \%$ to as low as $2.2 \%$ when stabilizers are placed and wired to the pectus support bar. ${ }^{2}$ Hebra et al. ${ }^{9}$ recommend a three-point fixation of the pectus bar, with an additional suture passed into the right hemithorax lateral to the sternum, and attaching the bar to a supporting rib using a nonabsorbable suture. Uemura et al. ${ }^{8}$ developed and reported a modification in 2003 for additional stabilization of the Nuss bar by wiring the bar directly to the ribs laterally using surgical wire, noting a decrease in bar movement; however, in the teenage population, they still noted a $27 \%$ incidence of bar movement. In 2004 Park et al. ${ }^{7}$ first reported their success in decreasing bar movement to $3.4 \%$ with a five-point wire fixation technique. An updated series of the same technique by Park et al. ${ }^{10}$ was reported with $3.6 \%$ occurrence of bar movement; none in 142 consecutive patients had movement when wire was used to affix the bars, but 2 patients had bar displacement when absorbable suture was used. Prior to our current experience with placement of two bars at the initial procedure, no previous series has reported no bar movement episodes.

Many authors have suggested using two bars in the certain patient populations, but no one to date has reported this as standard practice. In the original 1998 report by Nuss et al. ${ }^{1}$ they stated that "two bars are more effective than a single bar, but may cause over correction in some patients." Additionally, they stated that patients with certain connective tissue disorders may have a better result if two bars are placed. ${ }^{1}$ Uemura et al. ${ }^{8}$ recommended use of two bars in the teenage patient population, as this age group has more reported episodes of bar movement. Ong et al. ${ }^{11}$ reported overall good results in the teenage population with a single bar and lateral stabilizers, without using additional wire stabilization, but found $10.3 \%$ incidence of bar displacement. Yoon et al. $^{12}$ reported an overall incidence of $4.5 \%$ bar movement requiring reoperation in the late adolescent and adult age groups by application of a "compound bar" and narrowing the intercostal space with wires and a three-point bar fixation, as well as elevation of the sternum using a "Crane" technique and occasional insertion of double bars.

In our institution, we aim for repair of pectus excavatum at the beginning of puberty to take advantage of the remaining chest wall pliability, but avoiding the complication of recurrence after bar removal that is sometimes seen after a major growth spurt if the bars are placed at too young an age. The majority of patients with bar movement were in the teenage population, although 1 patient was approximately 6 years old and another almost 25 years old.

However, in evaluating our data after routine single-bar placement, we were dissatisfied with the $15.5 \%$ rate of bar movement. These episodes of bar displacement were equally distributed over the years 2000-2006 and therefore are not attributable to learning the surgical procedure (Fig. 2). After we reviewed the available literature, in November 2006, it

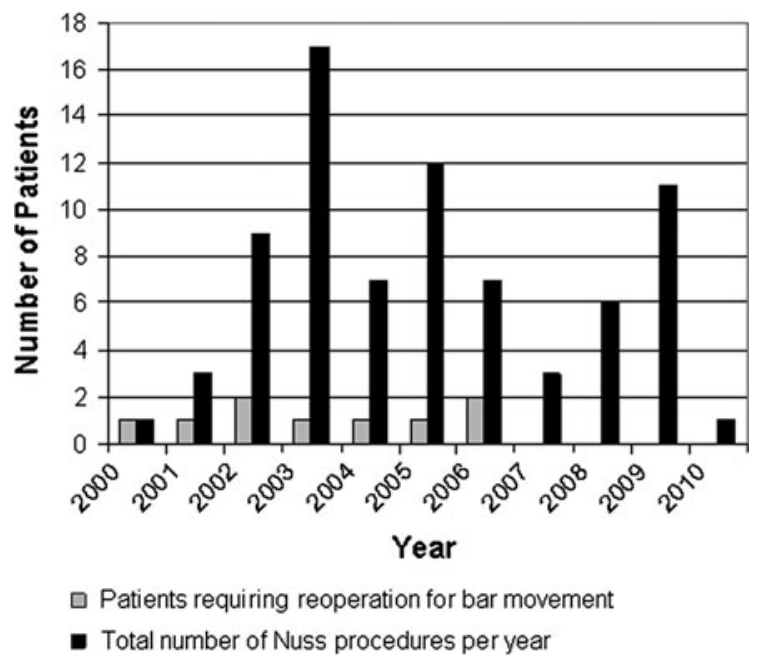

FIG. 2. Bar movement episodes per year, as a function of number of procedures.

became our standard practice to place two pectus support bars at the initial operation, in an attempt to decrease the troublesome complication of bar movement requiring operative revision.

It has been demonstrated that it takes $32.5-41.2$ pounds of force necessary to elevate the sternum in patients 11-19 years of age, unrelated to severity of Haller index unless the index is over 5.0. ${ }^{13}$ Since the inception of two-bar initial placement, we have had no episodes of bar dislodgement. We believe this increased bar stability to be due to better distribution of the force across two bars, minimizing the force or imbalance necessary for any one bar to bear.

Although historical bias certainly exists in any retrospective review, nothing in our operative technique, antibiotic regimen, or postoperative routine changed during the transition from the standard initial one- to two-bar insertion. Our mean patient age did not differ significantly between groups, nor did the severity of the defect as determined by the Haller index. Additionally, the hospital length of stay did not differ significantly between groups, demonstrating that the placement of a second bar did not affect the overall postoperative course. The possibility of increased wound complications from a larger subcutaneous dissection was evaluated, and no significant difference was noted in the rate of wound infection between groups. Total operating room time and case time were slightly and significantly longer in the two-bar group compared with the one-bar group. However, we believe this additional initial procedure time, approximately 18 minutes, to be time well spent in preventing an additional operation due to bar displacement and therefore of little clinical relevance overall. It should be noted that at the time of bar removal, it is necessary to open both sides of the chest, as opposed to just a single side as is performed when only one lateral stabilizing plate is used. This is still performed as an outpatient procedure and has not been found to be of significant morbidity.

By straddling the deepest point of the pectus defect with two support bars, we believe this distributes the force more effectively, thereby placing less stress on a single bar and minimizing movement. Primary placement of two bars has now become standard practice in our institution for correction of pectus excavatum by the Nuss procedure and would be our 
recommendation for consideration by other centers, as we believe this may be beneficial in preventing bar movement requiring reoperation.

\section{Disclosure Statement}

No competing financial interests exist.

\section{References}

1. Nuss D, Kelly RE, Croitoru DP, Katz ME. A 10-year review of a minimally invasive technique for the correction of pectus excavatum. J Pediatr Surg 1998;33:545-552.

2. Goretsky MA, Kelly RE Jr, Croitoru D. Chest wall anomalies: Pectus excavatum and pectus carinatum. Adolesc Med 2004; 15:455-471.

3. Vegunta RK, Pacheco PE, Wallace LJ. Complications associated with the Nuss procedure: Continued evolution of the learning curve. Am J Surg 195:315-317.

4. Nuss D, Croitoru DP, Kelly RE Jr. Review and discussion of the complications of minimally invasive pectus excavatum repair. Eur J Pediatr Surg 2002;12:230-234.

5. Marusch F, Gastinger I. Life-threatening complication of the Nuss-procedure for funnel chest. A case report [in German]. Zentralbl Chir 2003;128:981-984.

6. Nuss D, Kelly RE, Croitoru DP, Swoveland B. Repair of pectus excavatum. Pediatr Endosurg Innovat Techn 1998;2: 205-221.

7. Park HJ, Lee SY, Lee CS, Youm W, Lee KR. The Nuss procedure for pectus excavatum: Evolution of techniques and early results on 322 patients. Ann Thorac Surg 2004;77:289-295.
8. Uemura S, Nakagawa Y, Yoshida A, Choda Y. Experience in 100 cases with the Nuss procedure using a technique for stabilization of the pectus bar. Pediatr Surg Int 2003;19:186-189.

9. Hebra A, Gaudereer MWL, Tagge EP, Adamson WT, Othersen HB Jr. A simple technique for preventing bar displacement with the Nuss repair of pectus excavatum. J Pediatr Surg 2001;36:166-168.

10. Park HJ, Seock YL, Lee CS. Complications associated with the Nuss procedure: Analysis of risk factors and suggested measures for prevention of complications. J Pediatr Surg 2004;39:391-395.

11. Ong CCP, Choo K, Morreau P, Auldist A. The learning curve in learning the curve: A review of Nuss procedure in teenagers. ANZ J Surg 2005;75:421-424.

12. Yoon YS, Kim HK, Choi YS, Kim K, Shim YM, Kim J. A modified procedure for late adolescent and adult pectus excavatum. World J Surg 2010;34:1475-1480.

13. Fonkalsrud EW, Reemtsen B. Force required to elevate the sternum of pectus excavatum patients. J Am Coll Surg 2002;195:575-577.

Address correspondence to:

Amy B. Stanfill, MD

Department of Pediatric Surgery Children's Hospital of Illinois

University of Illinois College of Medicine at Peoria 420 NE Glen Oak Avenue, Suite 201

Peoria, IL 61603

E-mail: amy.b.stanfill@osfhealthcare.org 